\title{
The variety of sterility and gradual progression to fertility in hybrids of the horse and donkey
}

\author{
E. Zong and \\ G. Fan
}

Lanzhou Research Institute of Animal Science, Chinese Academy of Agricultural Science, Lanzhou, Gansu, China.

Generally speaking, the majority of $\mathrm{F}_{1}$ hybrids between the horse and donkey are sterile and do conform to the hypothesis of gametogenic breakdown. The results of our own research, nevertheless, lead us to conclude that there is variability in degree of sterility and fertility for both $F_{1}$ and $B_{1}$ individuals, with a gradual advance from sterility to fertility being seen. This is the subject we will discuss from the points of view of oestrus, ovulation, mating, pregnancy, spermatogenesis, endocrine activity, isozymes and karyotypic analysis in this paper.

\section{INTRODUCTION}

In general, mules (mare $\times$ jack donkey) and hinnies (jenny donkey $\times$ stallion) cannot reproduce, but occasionally, these $F_{1}$ hybrids do backcross with a stallion or jack donkey and give birth to a $B_{1}$ hybrid foal. The extreme rarity of such events leads most people, however, to believe that mules and hinnies are sterile hybrids. An hypothesis put forward early this century to explain this sterility suggests that mules and hinnies are allodiploids in which the chromosomes at meiosis are unable to complete their synapsis (Wodsedalek, 1916). Germ cells are thus unable to pass through gametogenesis to produce eggs or sperm. Anderson (1939) later suggested that a few fertile female mules did exist because they were able to produce chromosomally balanced ova containing only the chromosomes of the horse. The hypothesis was later extended by Chandley (1981) who suggested that in both the female mule and hinny, only the maternal chromosome set segregated into the ovum, those of the horse passing into the egg of the mule, those of the donkey passing into the egg of the hinny. The $\mathrm{B}_{1}$ offspring of a female mule and stallion, for example, would therefore be a horse.

Hybrid sterility is a wide-ranging problem in biology and a complete study has formed around it. Hence, any disagreement must be backed by extensive scientific fact. Because of this, we have put together all of our material to support our conclusions.

Limited by the length of the paper, we cannot explain certain facts in detail, and are forced to omit some detail explaining our methods.

\section{MATERIALS AND METHODS}

\section{Investigation of the oestrus rate in female mules}

Investigations of the oestrus rate of female mules (including hinnies) were made in the breeding seasons between 1954 and 1955. Among the 209 female mules examined, 33 were raised by farmers in the countryside, and the other 176 were used for city transportation.

\section{Oestrus, ovulation and mating of female mules and hinnies}

During 1954-56, we fed eight female mules and hinnies for the purpose of studying their oestrus, ovulation and mating. One mule and one hinny among them was fertile. The oestrus of the mules was investigated and noted down by a certain person at a particular time. Their ovulation was estimated by feeling their ovaries with a hand through the rectum. These mules were served by a stallion or a jack donkey. 


\section{Histological investigation of a female mule's ovary}

The ovary of a female mule was excised in the breeding season (June 1955) when the mule was 4 years of age. We excised a small section close to the surface of the ovary, fixed it in Bouin's solution, embedded it in paraffin, and stained it with haematoxylin and eosin.

\section{Oestrus, ovulation and mating of a $B_{1}$ foal from a female mule and stallion}

During the three years when the $\mathrm{B}_{3}$ was aged from seven to nine, ( $B_{1}^{\prime}$ table 2 ) it had no oestrus (its state before this period was unknown). The histologic section of its ovary was made in November, 1984 by the above-mentioned method.

\section{The collection and testing of a male mule's semen}

We collected the semen artificially from a male mule aged three years, then examined it under the microscope for the presence of sperm and to observe the shape of the sperm, if present.

\section{Histological investigation of the male mules' testes}

During 1954-85, histological sections of 20 male mules (including hinnies), aged $2 \cdot 5$ to 11 years, were made in the breeding season. Small sections close to the testis surface were fixed in Bouin's solution immediately after removal, embedded in paraffin, and stained with haematoxylin and eosin.

\section{Histological investigation of the testis of a $B$, male (female hinny $\times$ jack donkey)}

When $\mathrm{B}_{1}^{3}$ (table 2) was 32 months old (3 May 1983), we excised its left testis, and cut a small specimen close to the surface. It was fixed in Bouin's solution, embedded in paraffin, and stained with haematoxylin and eosin. When $B_{1}^{3}$ was 44 months of age ( 8 May 1984), we excised a small tissue section from its right testis, and treated it in the same way. Meanwhile we also made a smear and a squash preparation for chromosome observation.

After the excision of the small tissue section in $1984, B_{1}^{3}$ animal was given in injection of 2300 unit PMSG. Then 23 days later another tissue specimen was excised by the same method. Histological sections of the testes of another three-year-old $B_{1}$ of the same blood line (not listed in table 2), were made by Zhu Xuanren in December, 1956.

\section{Testing of the endocrine hormones of male $B_{1}^{3}$ (female hinny $\times$ jack donkey)}

Blood samples for testing were collected on alternate days, 14 times from the neck vein of the $B_{1}^{3}$ male. The collection was always made between 8-9 am, samples then being allowed to settle. The separated blood serum was stored at $-20^{\circ} \mathrm{C}$. After all collections were completed the total sample was sent to the Research Laboratory for Livestock Endocrine in the Northwest Agricultural University for radioimmunoassay.

\section{Determination of esterase isozymes}

Two $\mathrm{B}_{1} \mathrm{~s}$, one $\left(\mathrm{B}_{1}^{5}\right)$ a female, aged six months, the other $\left(B_{1}^{3}\right)$, a male, five years old were offspring of a female hinny $\times$ a jack donkey. Their blood serum was collected in the way described above, and then sent to the Gansu Agricultural University for isoelectrofocussing. Blood sera of a hinny and a donkey were used as controls.

\section{Making of chromosome preparations from horses, donkeys, mules, hinnies and $B_{1} s$}

Karyotypes of eight $B_{1} s$ and several horses, donkeys, mules and hinnies for comparison were made from lymphocytes following collection of blood from the neck vein. Standard culture procedures were used.

\section{The study of phenotype in $B_{1}$ animals}

We observed $12 \mathrm{~B}_{1} \mathrm{~s}$, had photographs of another four, and followed the progress of another eight for 2-6 years. Our purpose was to get to know their changing phenotypic features.

\section{RESULTS AND ANALYSIS}

\section{Oestrus behaviour in female mules and hinnies}

According to our investigations, all of the 33 female mules in Wushan County, Gansu Province showed oestrus behaviour in the breeding seasons, but cycles were irregular. Yet among the 176 female mules of the Lanzhou Transportation Team, only 
Table 1 The oestrus cycle and duration of oestrus in female mules and hinnies

\begin{tabular}{|c|c|c|c|c|c|c|}
\hline \multirow[b]{2}{*}{ Number } & \multirow{2}{*}{$\begin{array}{l}\text { Total number } \\
\text { of cycles }\end{array}$} & \multicolumn{2}{|c|}{ Oestrus cycle (days) } & \multicolumn{2}{|c|}{ Duration (days) } & \multirow[b]{2}{*}{ Note } \\
\hline & & Range & Mean & Range & Mean & \\
\hline 301 & 7 & $17-25$ & $21 \cdot 0$ & $3-9$ & $6 \cdot 5$ & Had a miscarriage in 1954 \\
\hline 302 & 8 & $18-22$ & $21 \cdot 2$ & $2-9$ & $6 \cdot 3$ & Gave birth to a $B_{1}$ in 1953 \\
\hline 303 & 7 & $21-25$ & $23 \cdot 3$ & $4-8$ & $6 \cdot 1$ & \\
\hline 306 & 11 & $14-26$ & $18 \cdot 5$ & $3-7$ & $4 \cdot 9$ & \\
\hline 307 & 5 & $16-23$ & $19 \cdot 3$ & $3-5$ & $4 \cdot 0$ & \\
\hline 308 & 2 & & & & $4 \cdot 0$ & Oestrus was disturbed \\
\hline Average & & & $20 \cdot 5$ & & & \\
\hline
\end{tabular}

half of them showed oestrus behaviour during the breeding season while the other half had none.

The cyclicity of city mules was even more irregular than that of the mules in the countryside. This disparity might be related to the difference in the amount of work done, and different foodstuffs consumed. The investigations of the country mules were made in slack farming seasons when the female mules had little work to do, most of them having grazed in the mountains where there was ample fodder grass. By contrast, the mules for city transportation worked heavily all the year round and had no fodder grass. This might affect their oestrus patterns.

\section{Oestrus, ovulation, mating and pregnancy of female mules}

The 59 oestrus cyles of eight female mules during two years are listed in table 1.

We bought these eight female $F_{1}$ s because they showed oestrus signs. Animal 301, a female mule, was mated with a stallion in 1953 by its original owner out of curiosity. The owner was not aware that the mule had become pregnant and used it for work as usual, causing miscarriage of the foetus. Animal 302 was a female hinny. It was always herded with jack donkeys and mating occurred frequently. In 1953 it bore a $B_{1}$ foal. These two $F_{1}$ hybrids were fertile. Their oestrus was regular. The others shared large dissimilarity between individuals. As for oestrus cycle length, the longest was 34 days, while the shortest was 13 days; the average being 20.5 days. On two occasions the interval between two cycles lasted 50 days. Such a phenomenon might be related to hormones. Of special importance here is that some bird hybrids may also share the phenomenon that indistinct secondary sexuality is concerned with endocrine conditions.

It has been proved by the 159 examinations of the 34 cycles of the six female mules that both the right and left ovaries of female mules can form ovarian follicles, and their production of follicles may not be alternate. The degree of ovarian follicle development before rupture is lower than that of the horse or donkey. Most ovarian follicles of mules and hinnies can burst, but those that cannot, and those that cannot be clearly judged, account for 27 per cent of the total number of cycles. This is rare in horses and donkeys. Rupture of the ovarian follicles in the female mule may be complete in one day or may last for 11 days in different individuals or in different cycles of the same individual. However, most of the ruptures occur in the period of 4-7 days. In the horse, rupture of the follicle means that ovulation has occurred. In female mules this conclusion cannot necessarily be reached.

The optimum time to mate a female mule is still unknown. Referring to information about the mating of horses and donkeys, and the rupturing of the follicle in the female mule, we decided to have the mules served once daily from day three of oestrus. For eight female mules in oestrus, 59 services were given by a stallion and a jack donkey of known normal semen quality. Although none of the mules bore offspring, two phenomena could be taken as signs that pregnancy had occurred: the first was that the next oestrus of some mules disappeared or appeared quite weak. The second was that a possible early embryo could be felt in the uterine horn at examination through the rectum of two mules 40-60 days after mating. This became smaller and totally disappeared later, perhaps indicating the end of development of a fertile egg. 


\section{Histological observation of the female mules' ovary}

We observed only three mules. In one of the ovaries, there existed a large follicle, the diameter of which was about $4 \mathrm{~cm}$. There were also some smaller follicles. At the microscopic level, we saw primary follicles, and follicles I and II, but no oocytes were found. A similar phenomenon was also reported by Benirschke and Sullivan (1966). Nevertheless it can be deduced that, from the birth of a $B_{1}$, and the growth of an embryo in the uterine horn after mating of a female mule, there is an undoubted ability to produce mature ova in some female mules. Bielanski and Zapletal (1968) have also reported finding oocytes in the oviducts of two female mules.

\section{Histological observation of the ovarian tissue of $B_{1}^{\prime}$ (female mule $\times$ stallion)}

A statement should be made first here: the $B_{1}$ had no oestrus cycles from 1981 to 1984 . The excision of its ovary took place in December, 1985 when the breeding season had finished and this almost certainly could have affected the development of its ovarian follicles. Yet in its ovary there existed three follicles of about $1 \mathrm{~cm}$ diameter. Moreover there were some follicles with diameters from 1 to $3 \mathrm{~mm}$. Under the microscope, primary follicles and follicles I and II were seen (fig. 1), but no oocytes were found. This was quite similar to the histological findings on the ovary of the female mule. This tells us that the characteristics of a hybrid in this $\mathrm{B}_{1}$ obviously existed. Cuenot (1947) has reported that all of three female $B_{1}$ s of such a blood line

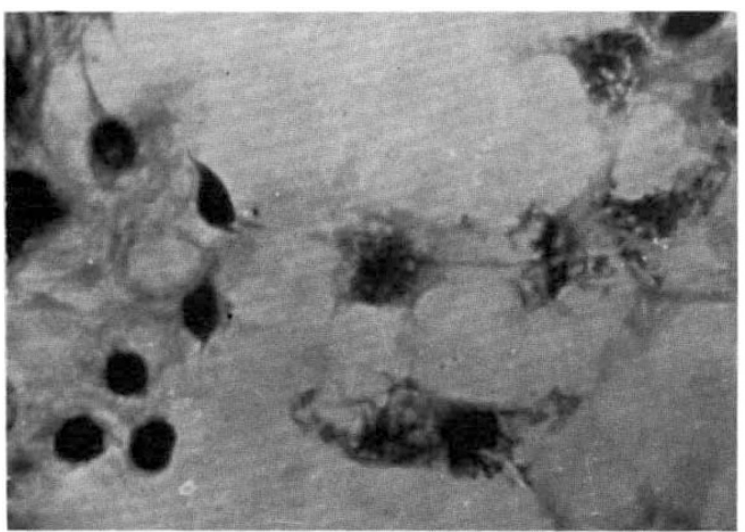

Figure 1 A section of ovary of $B_{1}^{1}$ (female mule $\times$ stallion). Follicles Stage I and II can be seen but there are no oocytes. Magnification $\times 66$. he has examined are fertile, though there exists dissimilarity between individuals.

\section{Seminal analysis on a male mule}

The male mule being examined was three years old. We gathered its semen nine times in the period from 25 May to 9 July 1955 . Five out of nine times sperm were found on the slides. On the smears there were from three to 90 sperm, the count being generally about 50 . But no motile sperm were seen. By comparison with sperm of the normal horse or donkey, most of the mule sperm were irregular in shape, e.g., their heads were rhomboid, the neck slim or tail long, etc. This result is, in the main, similar to that in the reports by Trujillo et al. (1969) and by Chandley et al. (1974).

\section{Histological observation on the mule testis}

In most seminiferous tubules, the number of spermatogonia was small, and the number of primary spermatocytes even smaller. Many seminiferous tubules contained none or only one or two primary spermatocytes. Only in a few seminiferous tubules of a specific mule testis did we find more primary spermatocytes, but they were much fewer in number than in the horse or donkey. However, dissimilarity exists in the degree of spermatogenic activity for individual mules and hinnies and we noted that more spermatocytes were present in those animals having sperm in their semen. In them, we also found groups of four round spermatids, bridges between the nuclei, and a few differentiating spermatids (fig. 2), but no spermatozoa were seen. In all the histologic sections of male mules'

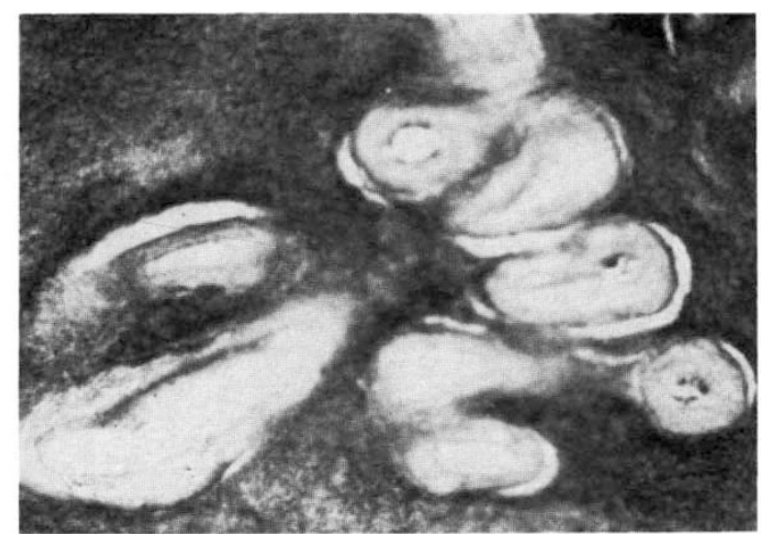

Figure 2 Four spermatid nuclei and intercellular bridges between them in the seminiferous tubules of a male mule. On the right is a spermatid. 


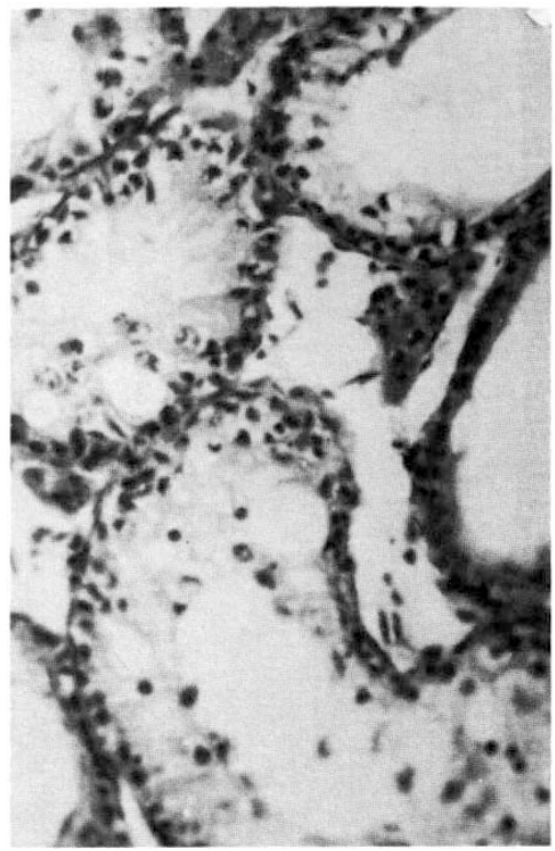

$a$

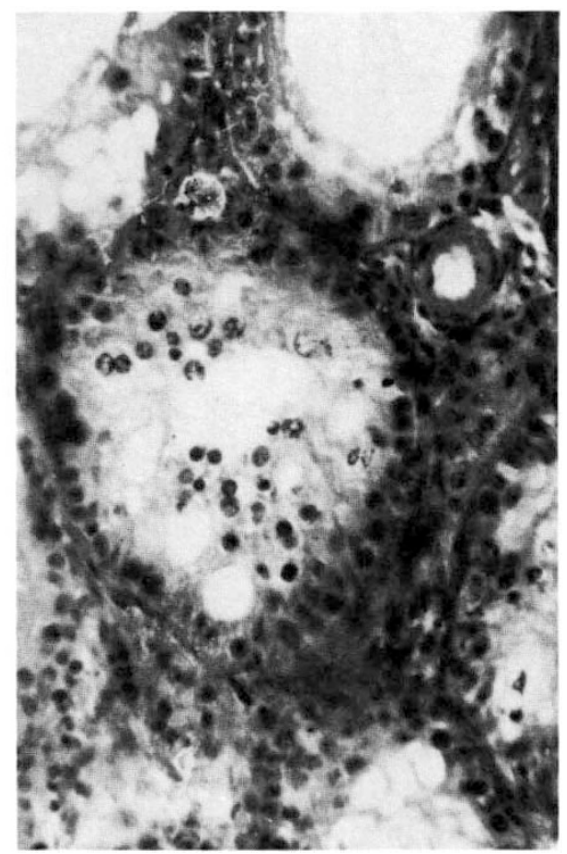

$b$

Figure 3 Seminiferous tubules. (a) Of a male mule. (b) Of a $B_{1}$ male (female hinny $\times$ jack donkey) born 1953 . In the latter, $1^{\circ}$ spermatocytes and a few spermatids are present.

testes, there must be primary spermatocytes that are degenerating and in these, meiotic chromosome aberrations can be seen. This is the specific evidence for the statement that allodiploidy is the basic reason for the hybrid sterility. However, dissimilarities may not all occur in a specific part of the process of meiosis, but they may exist in different individuals or in the different seminiferous tubules of the same individual, even in different cells.

\section{Histological observation on the testes of $B_{1}$ individuals}

Testis sections from two $B_{1}$ individuals were examined. Both were of the same blood line, and were the backcross offspring from a female hinny and a jack donkey. One (not listed in table 2) was born in 1953. Its testes were excised in October, 1956 the histological sections being made in December of the same year. The other $\left(B_{1}^{3}\right)$ was born in 1980, testis samples being taken on three occasions in 1983 and 1984. In the seminiferous tubules of the former $\mathrm{B}_{1}$, there were more spermatogonia and primary spermatocytes than in most mules. Furthermore a few secondary spermatocytes were found (fig. 3 ) but there were no sperm. In 1955, before the testes were biopsied, we had collected semen artificially, but no sperm were found on that occasion either.

In the seminiferous tubules of $\mathrm{B}_{1}^{3}$, born in 1980 , primary spermatocytes were more common than in the average male mule or in the $\mathrm{B}_{1}$ born in 1953 , and a few secondary spermatocytes and spermatids were seen (fig. 4). In $B_{1}^{3}$ changes in the testis with age and after injection of PMSG were also recognized. When the $B_{1}$ was 32 months old, no sperm were found, though there were many primary spermatocytes, a few secondary spermatocytes and spermatids, and even metamorphosed spermatids with lengthened nuclei. The testis at 44 months showed better spermatogenic development than at 32 months, the increase occurring not only in the number of the above-mentioned cells, but also in the number of metamorphosed spermatids (fig. 5). These changes occurring with age may relate to many factors, indicating that the cause of hybrid sterility is not as simple as people usually believe. Furthermore, in the testis section excised from this $\mathrm{B}_{1}$ animal 23 days after PMSG injection, primary spermatocytes became less in number while spermatids, including some metamorphosed spermatids with Golgi acrosomic granules increased, and some deformed spermatozoa could be seen 
(figs 6 and 7). As the choice of dose and time of injection on this one $B_{1}$ animal may not have been optimal, it cannot be concluded that the changes seen were significant, but the appearance of spermatids with Golgi acrosomic granules and of deformed spermatozoa should not be overlooked as a contribution to the study of hybrid sterility.

\section{Endocrine hormones in male $B_{1}^{3}$}

Endocrine hormones have a close relationship with breeding and hormonal disturbance can cause the sterility of both male and female livestock. Superovulation may be induced by an injection of endocrine hormones, this kind of superovulation actually causing meiosis. One of the functions of PMSG is to promote the spermatogenesis of male animals. Based on this we measured the amount of testosterone and 17- $\beta$ estradiol in the blood of the $B_{1}^{3}$ male born in 1980 before removal of the testis specimen. The results showed us that the amount of testosterone in the $B_{1}$ was 505 times more than that of a donkey assayed at the same time, while the $17-\beta$ estradiol of the donkey was 2.5 times more than that of the $B_{1}$ (both were measured with the radioimmunoassay (RIA). Compared with the donkey, these findings were somewhat irregular, but they could still have an affect on spermatogenesis.

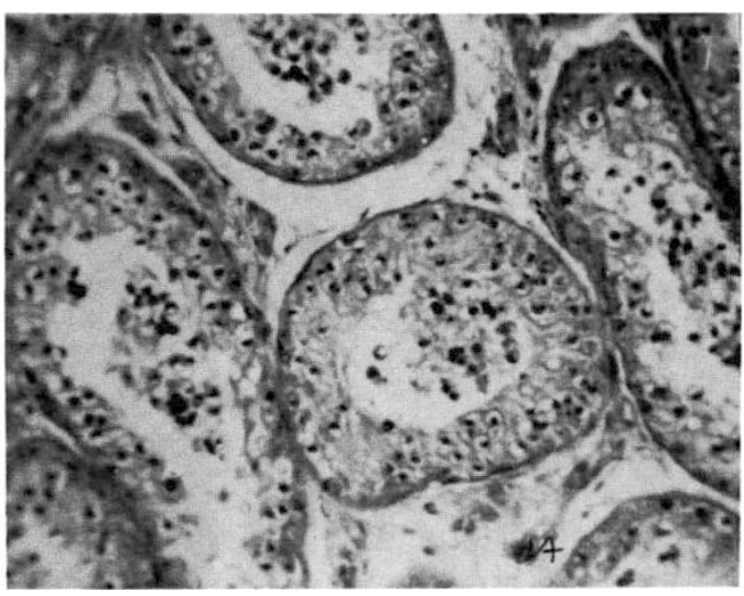

Figure 4 Numerous primary spermatocytes and a few sper matids in the seminiferous tubules of $B_{1}^{3}$ (female hinny $x$ jack donkey) born in 1980 , when 2.5 years old.

\section{The estimation of the esterase isozymes of the two $B_{1}$ males $B_{1}^{3}$ and $B_{1}^{5}$ (female hinny $\times$ jack donkey)}

According to the study of Zhang Deshou (1987) the blood serum of a horse contains esterase isozymes, as does that of the mule and hinny. The donkey however, contains none. The interesting

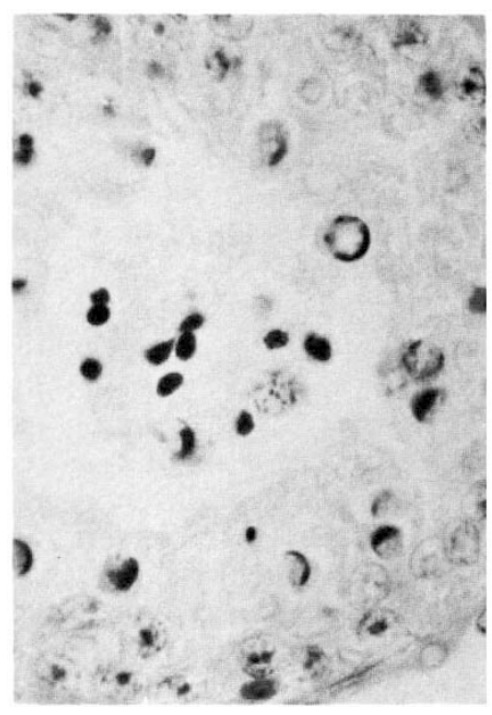

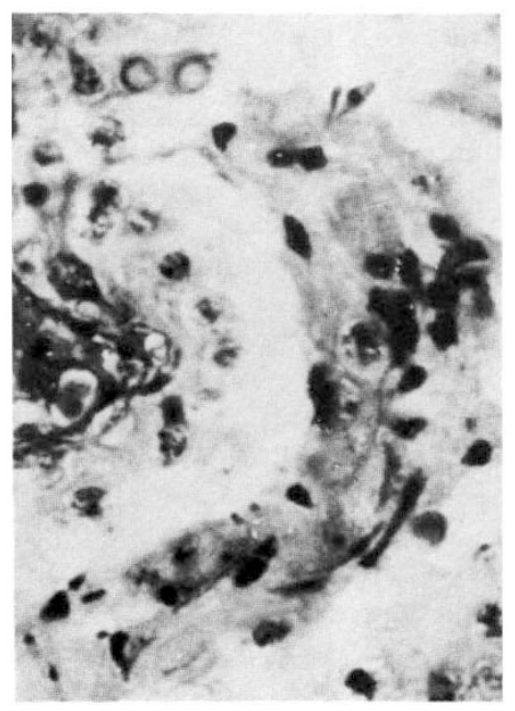

$b$

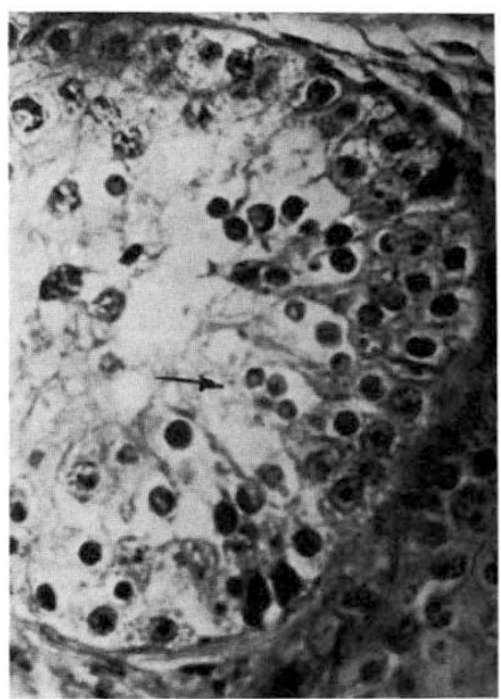

$c$

Figure 5 (a) Elongating spermatids in the testis of $B_{1}^{3}$ age 32 months. (b) The same $B_{1}$ testis seen at 44 months. Some spermatids now show the early sperm head shape. (c) The same as (b). The arrow points to four spermatid nuclei which have not completely divided at meiosis. 


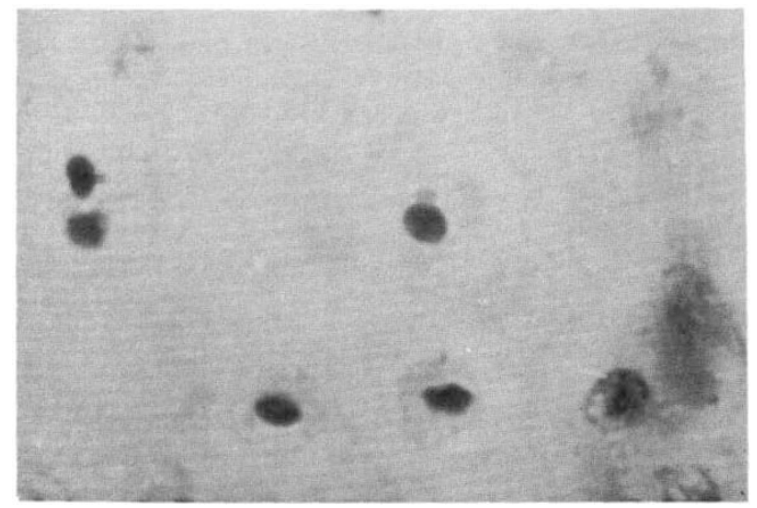

Figure 6 Differentiating spermatids with Golgi acrosomic granules which appeared in $\mathrm{B}_{1}^{3}$ (female hinny $\times$ jack donkey) following an injection of PMSG given at 44 months of age.

result was that while one $B_{1}$ male showed the isoenzyme, the other did not (fig. 8).

\section{Phenotype of $B_{1}$ animals}

Between 1953 and the present time, the phenotypes of $13 B_{1}$ animals have been studied. Three (including $B_{1}^{1}$ and $B_{1}^{2}$ ) were offspring of female mules crossed with stallions and their phenotype resembled that of the horse (figs $9,10,11$ ). The early hypothesis that the offspring of a female mule and a stallion is a horse, may be based on this kind of observation. Four $\mathrm{B}_{1}$ offspring (including $\mathrm{B}_{1}^{6}$ and $B_{1}^{7}$ ) were produced out of crosses between female mules and jack donkeys. Their phenotypes were quite variable. Those illustrated in figs 12 and 15 are much like donkeys; the one in fig. 13 looks more like a mule. The phenotype of the $B_{1}$ in fig. 14 which was only 6 months old, was still changing, but it resembles much more a donkey. The phenotypes of these four $B_{1} s$ are not typical mule. Their characteristics are of the backcross to a jack donkey. The remaining four $B_{1} s$ are the offspring of female hinnies and jack donkeys $\left(\mathrm{B}_{1}^{3}, \mathrm{~B}_{1}^{4}, \mathrm{~B}_{1}^{5}\right)$. Since Anderson (1939) put forward his theory that the offspring of a female mule and a stallion was a horse, some have suggested that the offspring of female hinnies and jack donkeys should be donkeys (Chandley, 1981). Yet our findings indicate that this may not be the case (figs 16-19). No matter what the shape of the head, the body, or the hair colour of the $B_{1}$ shown in fig. 18 , it shares the characteristics of the mule, and although the $B_{1}$ in fig. 16 has fewer characteristics of a mule than the $B_{1}$ in fig. 18 , it could not be mistaken for a donkey. The hair colour of the $B_{1}$ in fig. 17 looks like that of a donkey (no mule has this), but its hooves, limbs, mane, and the combination of cervical and thoracic vertebrae all resemble those of a mule. So these $\mathrm{B}_{1} \mathrm{~s}$, with no doubt, are still hybrids, and have not regressed to pure donkey. Those who hold that a female hinny and a jack donkey will give birth to a donkey may not have
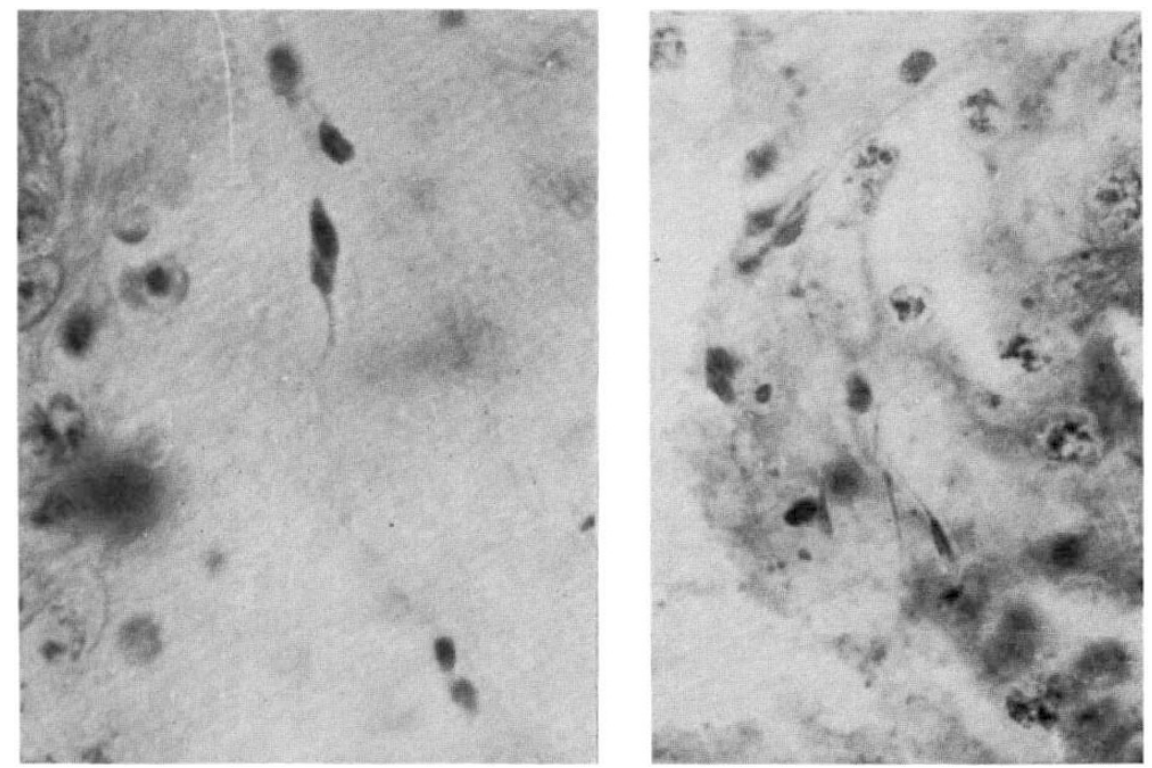

Figure 7 Deformed sperm in the tubules of $B_{1}^{3}$ (female hinny $\times$ jack donkey) following PMSG injection. 


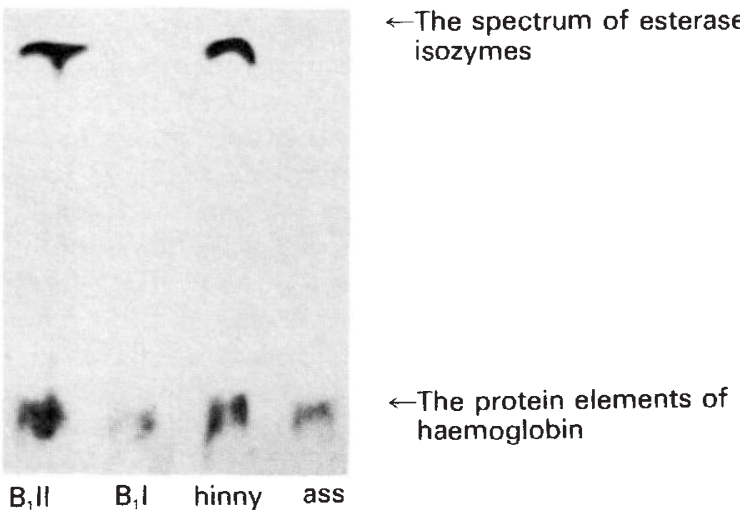

Figure 8 Isoenzyme spectrum of a hinny, a donkey and two $\mathrm{B}_{1} \mathrm{~s}$ made by isoelectro-focusing. $+\mathrm{B}_{1} \mathrm{I}$ (female hinny $\times$ jack donkey) was born in 1986; o $\mathrm{B}_{1}$ II (female hinny $\times$ jack donkey) was born in 1980 . The control hinny was the mother of $\mathrm{B}_{1} \mathrm{I}$; the control donkey its father.

seen this kind of $B_{1}$. According to the phenotypes of the $B_{1}$ s from these three kinds of backcross, the $\mathrm{B}_{1} \mathrm{~S}$ from different hybridizing combinations always have their own characteristics, and individuals of the same combination also have dissimilarities. There exist apparently varieties in them.

\section{Karyotypic investigations in $B_{1}$ animals}

During the period from 1981 to 1986 , we studied the karyotypes of eight $B_{1} s$, with karyotypes of several horses, donkeys, mules and hinnies for comparison. They are listed in table 2 .

In table 2 , the eight $\mathrm{B}_{1} \mathrm{~s}$ are divided into three groups according to their blood line, i.e., two ( $\mathrm{B}_{1}^{1}$ and $\mathrm{B}_{1}^{2}$ ) derived from female mule $\times$ stallion crosses, two $\left(B_{1}^{6}\right.$ and $\left.B_{1}^{7}\right)$ from female mule $\times$ jack donkey, and three $\left(\mathrm{B}_{1}^{3}, \mathrm{~B}_{1}^{4}\right.$ and $\left.\mathrm{B}_{1}^{5}\right)$ from female hinny $\times$ jack donkey. In the eighth case $\left(B_{1}^{8}\right)$, it was not known whether the dam was a mule or a hinny, but the sire was a donkey (fig. 20).

Chromosome counts of the $B_{1}$ progeny show that the ova from which they were derived had undergone reduction in the dam. Yet no two karyotypes were the same. With the exception of one of the $B_{1}$ progeny $\left(B_{1}^{2}\right)$ born to a female mule served by a stallion which appeared to show the karyotype of a horse, all the other seven karyotypes were different among themselves and different from those of the horse, donkey or mule. In the karyotype of $B_{1}^{1}$ (female mule $\times$ stallion) shown in fig. $21 \mathrm{~b}$ and $22 \mathrm{~b}$, there are 30 non-acrocentric and 32 acrocentric autosomes; this compares with counts of 48 non-acrocentric and 12 acrocentrics for the donkey and 26 non-acrocentric and 36 acrocentrics for the horse. (Eldridge and Blazak 1976 and our own observations.) The non-acrocentric chromosomes of $\mathrm{B}_{1}^{3}$ were 38 in number and the acrocentrics 22 (fig. 23), not the same as for the donkey. The remaining $B_{1}$ progeny are not described.

Since the contribution coming from the sire (horse or donkey) to the $\mathrm{B}_{1}$ progeny is known, any dissimilarity in the karyotypes must arise via the ovum of the mule or hinny female parent and it

Table 2 The Karyotypes of Horses, Donkeys, Mules, Hinnies and $\mathrm{B}_{1} \mathrm{~s}$

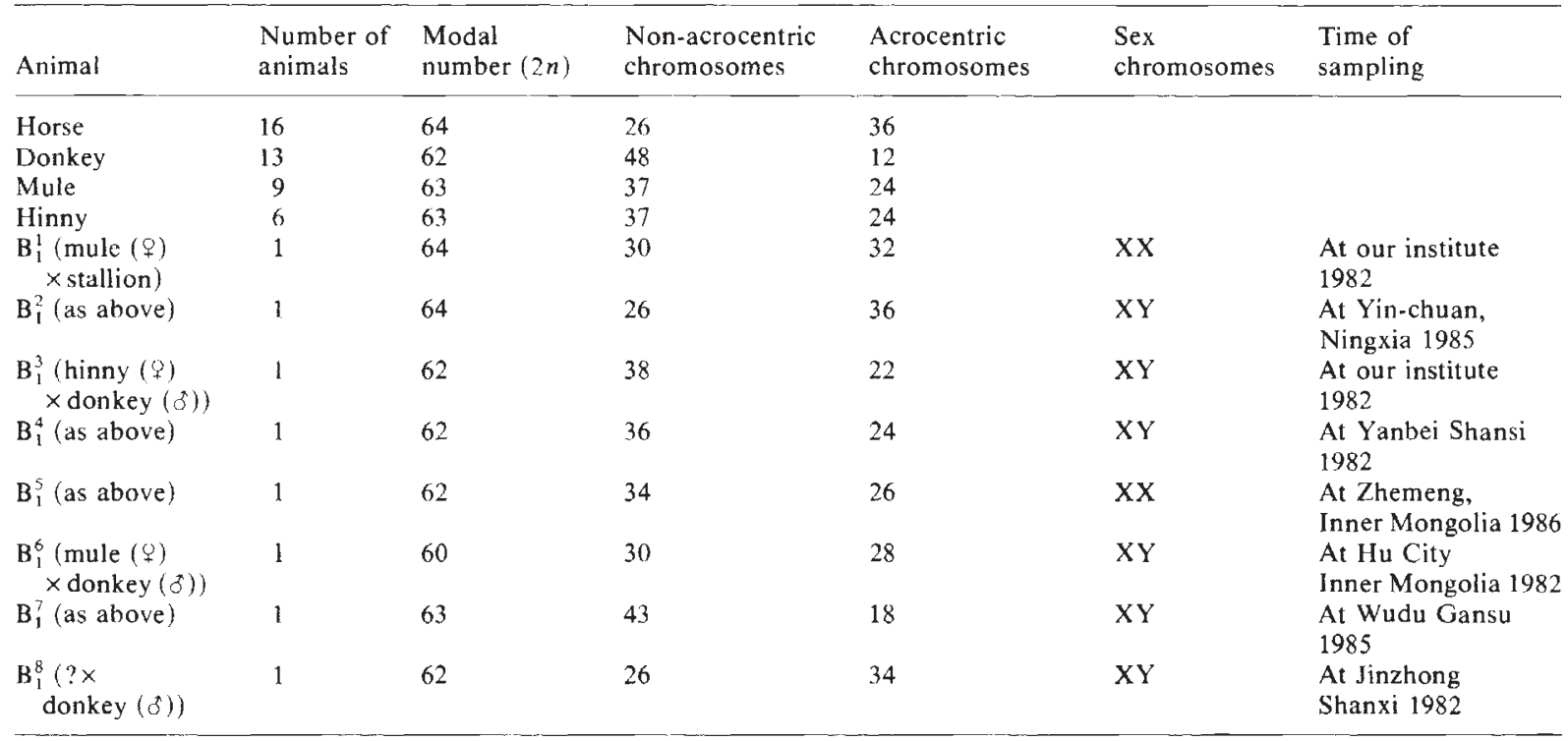




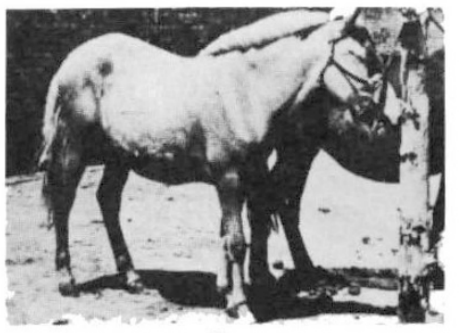

9

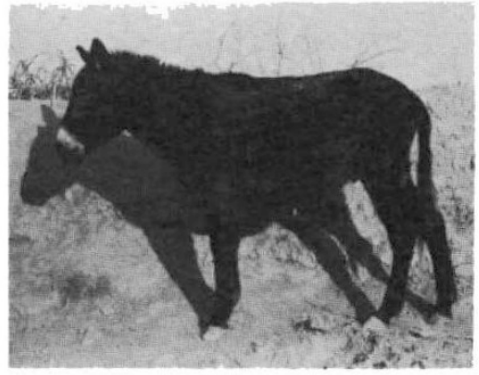

11

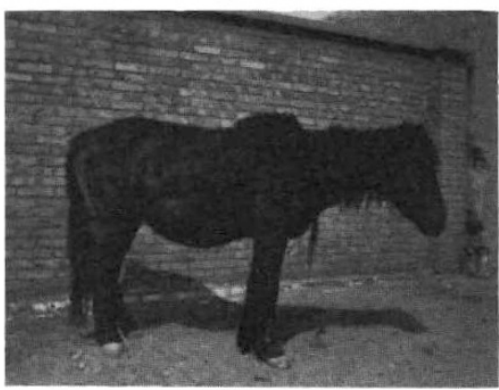

13

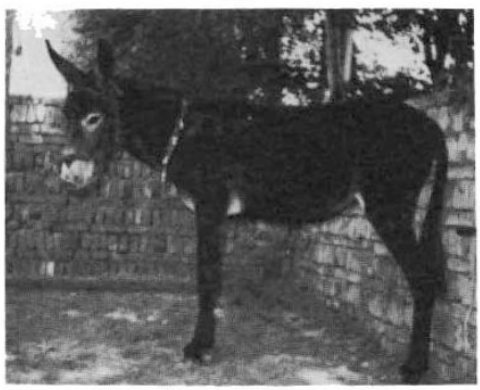

15

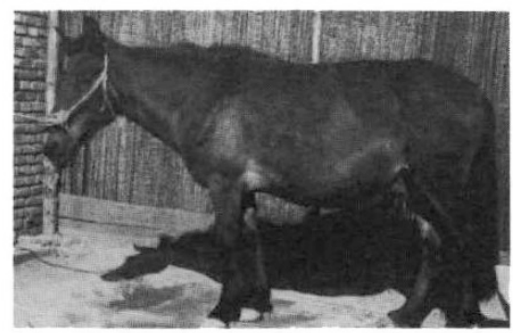

10

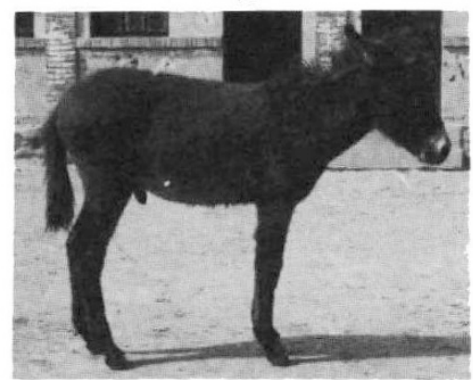

12

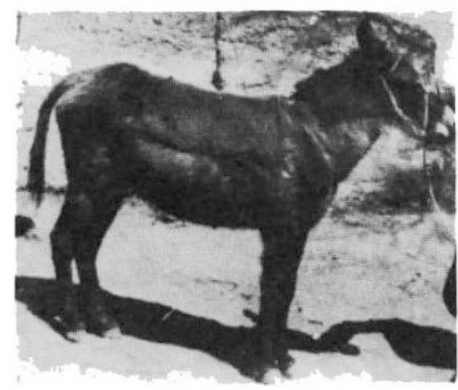

14

ies of the $B_{1}$ animals. Figures 9-11: Offspring of female mules and stallions. Figures 12-15: Offspring of Ick donkeys.

can be deduced from the $B_{1}$ chromosome counts that reduction has occurred at meiosis in the ovum. Nevertheless, our chromosome findings for the $\mathrm{B}_{1}$ progeny appear to show that in seven of the eight animals, a mixture of horse and donkey chromosomes have been inherited via the ovum. The exception to the rule is $\mathrm{B}_{1}^{2}$ born to a female mule and stallion, which showed the karyotype of a pure horse. This $B_{1}$ would appear to have arisen by segregation of a whole set of horse chromosomes into the mule egg as predicted by Anderson (1939) and Chandley (1981).

\section{DISCUSSION}

Analysis of the above results leads us to conclude that the sterility of mules and hinnies is not 

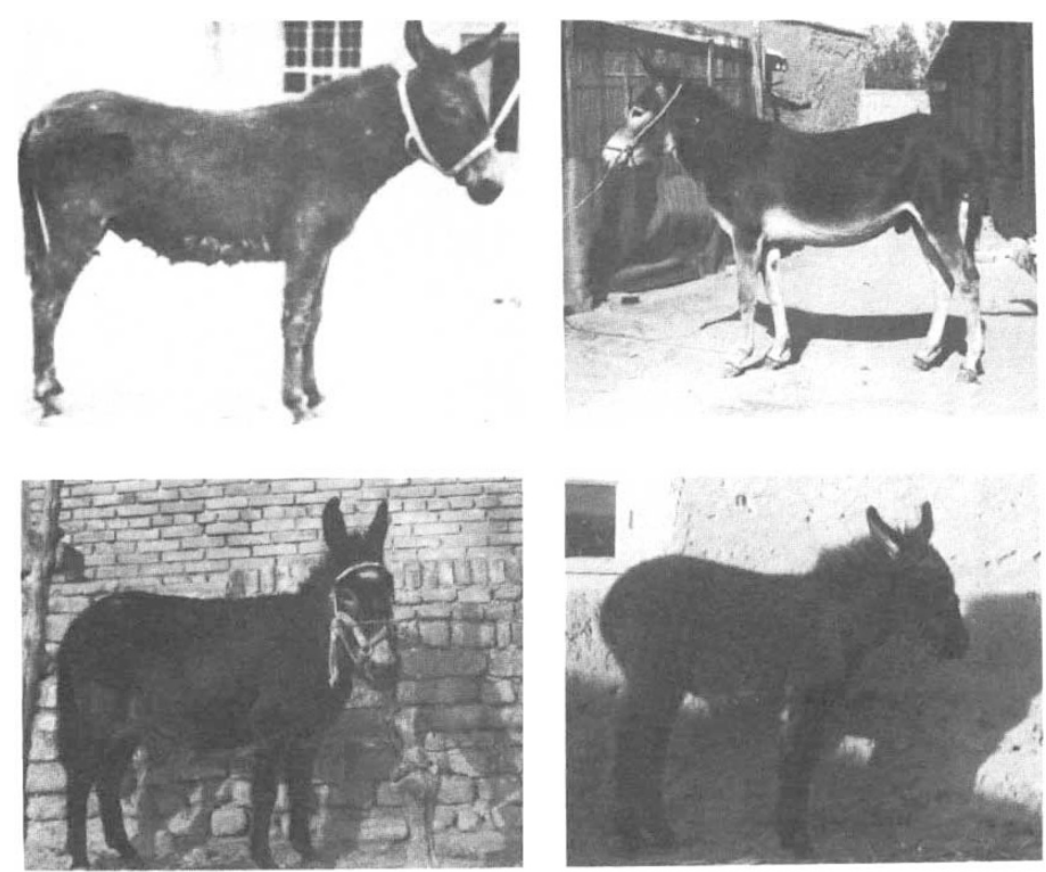

Figures 16-19 Offspring of female hinnies and jack donkeys.

absolute. While most $F_{1} s$ are sterile, a few female hybrids can sometimes bear a backcross on mating to a stallion or jack donkey. The proportion of such fertile $F_{1} s$ is small, but their fertility is indeed a genuine phenomenon.

We believe that the usual statement on sterility concerning mules and hinnies concentrates too

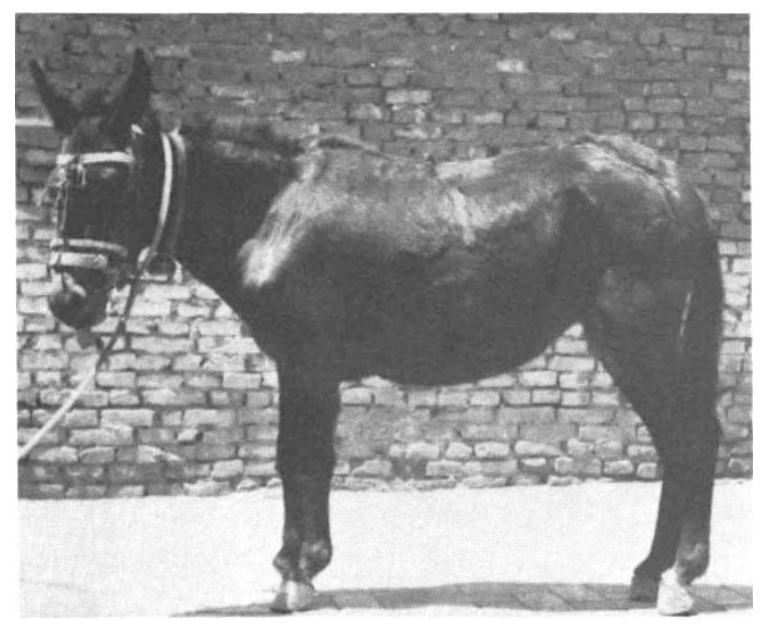

Figure 20 Offspring of mule or hinny female and jack donkey $\left(\mathrm{B}_{1}^{8}\right)$. much on cytological principles, many believing that sterility is a consequence of the cytological rules, and that fertility is absolutely impossible. However, some very well documented evidence of fertility has appeared (Rong et al., 1985, 1988; Ryder et al., 1985), since our own claim was made (Zong, 1983).

The hypothesis that the ova of fertile female mules and hinnies contain only a complete set of maternal chromosomes (Anderson, 1939; Chandley, 1981) provides one possible explanation for the production of a genetically balanced offspring to a fertile hybrid, but it does not deal with the question of the cause of fertility versus sterility. We believe that a new approach to the understanding of equine hybrid sterility is required.

The results of our research tell us that the so-called sterility of mules and hinnies varies in the way it manifests itself and the degree to which it occurs among different individuals. This is also true of the $B_{1}$ progeny which are born to a few female mules and hinnies backcrossed to the stallion or jack donkey. If one arranges these various phenomena systematically, the tendency of a gradual advance from sterility to fertility comes to light. For example, among all female mules and 


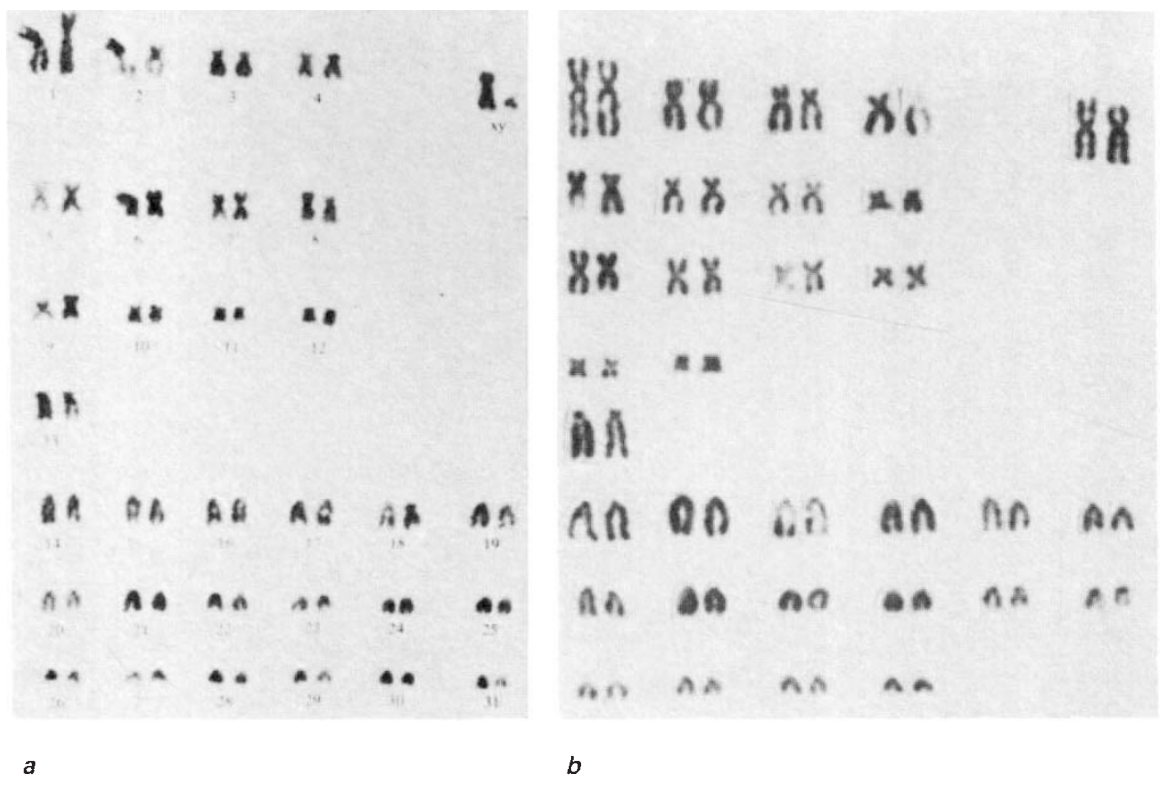

Figure 21 (a) Karyotype of the horse. From Hare and Singh (1979). (b) Karyotype of female B! (female mule $\times$ stallion). The animal looks like a horse.

hinnies, some never have oestrus, while some have it occasionally. There are some which cycle frequently but irregularly, and some which cycle quite regularly. Generally speaking, oestrus means that there must be an ovarian follicle under development in an ovary, and that ovulation will occur, yet this general biological rule varies greatly in mules. Some have apparent oestrus signs, but their follicles are far from fully developed, some do not ovulate though their follicles have developed well, some do not get pregnant even if they are mated again and again after rupture of

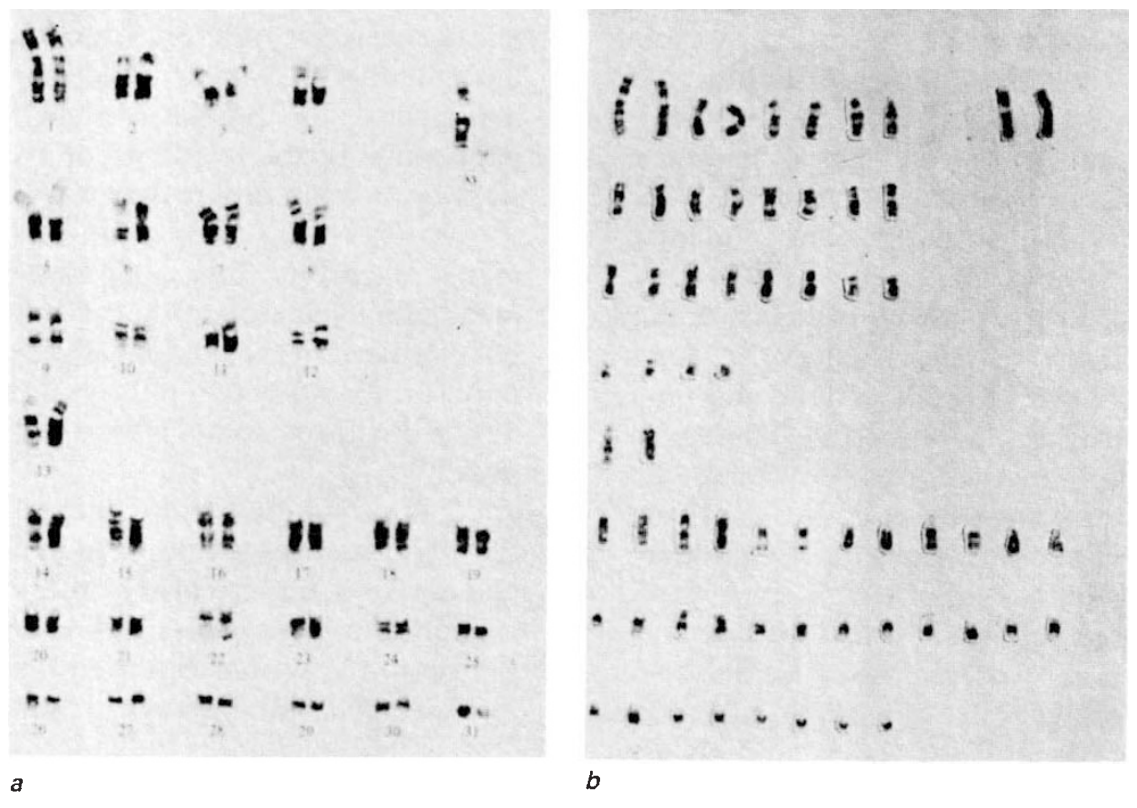

Figure 22 G-banded karyotypes of (a) the horse and (b) the $B_{1}$ female. 


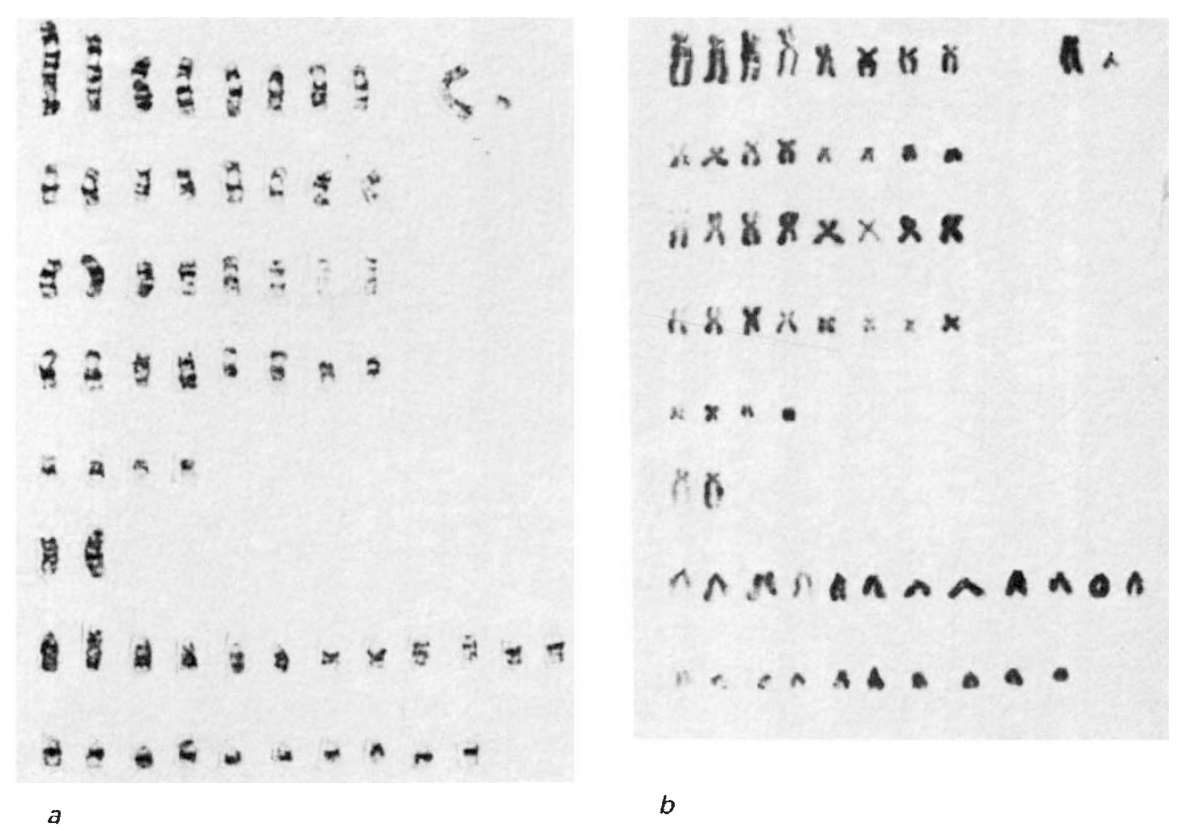

Figure 23 Karyotypes of $\mathrm{B}_{1}^{3}$ male (female hinny $\times$ jack donkey) (a) G-banded (b) Conventional staining.

their ovarian follicles. Some have embryos in their uterine horns after they have been mated, but these disappeared at examination 60 days later.

In our investigations, five female mules aborted at the late stages of pregnancy. Among female mules that can give birth, some never get pregnant again after the first delivery and some give deliveries one after another. One female hinny and one female mule which we bought and raised after they had produced offspring are examples. The mule had aborted in the later period of its pregnancy. Though they were mated again and again in two breeding seasons, the mule had a developing embryo at its uterine horn, which disappeared later. By contrast, Cuenot (1947) reported a female mule which gave birth to its second offspring after a few years interval. Moreover in our investigations we have also found one mule that gave two deliveries in the space of three years at Fengyi, Yunnan Province, China, 1954, and one that bore three offspring in a period of four years (among the three, one was aborted), at Quinglong, Hebei Province, China, 1954.

Various degrees of fertility also exist in male mules and hinnies. We have seen in the seminiferous tubules of the testes of more than 20 mules that most of the tissue of the testes contains very few primary spermatocytes. But there exist a few male mules and male hinnies that can produce a few spermatozoa (Trujillo, 1969; Chandley et al.,
1974). Until now, there is still no report of a fertile male mule, but such a possibility might exist. The unfortunate thing is that most male mules are gelded in their early years, and thus have no opportunity to breed. It is possible that a variety of sterility and a gradual advance to fertility may exist also in the male.

Study of the $B_{1}$ progeny shows that spermatogenesis for different individuals of the same bloodline is dissimilar. Also the level of spermatogenesis in $B_{1}$ males is higher than that of the male mule, further evidence of a variety of hybrid sterility and the progressive advance to fertility.

Variety is also reflected in the different assortments of chromosomes $(n)$ contained in the ova of different female mules and hinnies. This could be important in the gradual advance to fertility but only further study will tell us what kind of combination of chromosomes is required to make fertility possible.

This variety of sterility and gradual advance to fertility does not only occur in hybrids from horses and donkeys, but can also be found in other organisms such as birds, fishes and insects. For example, Lancetild (1929) has reported that the testis sizes of Drosophila $\mathrm{B}_{1}$ hybrids are different and suggested that a gradual advance from sterility to fertility occurred with this change. Dobzhansky (1936) further showed that variety and this gradual advance were related to the different combinations 
of allodiploid chromosomes. Other such examples exist in mammals, e.g., among the first generation of Java Bos gaurus $\times$ Bos taurus, males are sterile, while females can backcross with any one of their parental generation and give birth to $B_{1}$ progeny. Two out of twelve $B_{1}$ males recovered their fertility. Only 30 per cent of their spermatozoa were abnormal. Further backcrossing produced nine $\mathrm{B}_{2}$ male progeny, one of which had all normal spermatozoa and normal fertility. Zhang Song-zong (1986) has pointed out that hybridization of the four different combinations of Anas domestica genera resulted in different levels of hybrid sterility. Also, different individuals in the same combination could show great variability in the development of the reproductive system. In the testis of one out of the 37 "semi-Cairina moschata domesticus" (Jinding Anas (\%) $\times$ Cairina moschata domestica (ठ) spermatids in metaphase II were found. Su Dongdun et al. (1986) mated Cairina moschata domestica with Gaoyou Anas, and obtained fertile hybrids and went on to produce $\mathrm{F}_{4} \mathrm{~s}$. Cheng Guangchao (1986) made cytogenetic studies on the $F_{3}$ and proved that of their two sex chromosomes, one came from Gaoyou Anas and one from Cairina moschata domestica. This result is directly against Haldane's law, which states that among first generation hybrids whether male or female, if they are inviable, rare or sterile, their sex chromosomes will belong to the heterogametic sex.

An answer still has to be found to the problem of why there should be such varying degrees of $F_{1}$ hybrid sterility and progressive advance to fertility. Chromosomally, all karyotypes of $F_{1}$ s are the same except for the combination of $\mathrm{X}$ and $\mathrm{Y}$ in the male. The gradual change to a fertile state underlies the potential breeding capacity of mules and hinnies, and could form a theoretical basis for overcoming hybrid sterility. This could be of practical importance in the crossbreeding of animals. A good example of this is the following: Male $F_{1}$ hybrids of the cross American Bos gaurus (Buffalo) served by Bos taurus are sterile, while female $F_{1}$ hybrids are fertile. $B_{1}$ progeny born to these female $F_{1}$ hybrids, when backcrossed to their parental generation, show the same breeding capacity as the $F_{1}$ female parent.

These female $B_{1}$ s further backcrossed to males of the pre-parental generation, give a few male $B_{2} s$ which are fertile. These fertile $\mathrm{B}_{2} \mathrm{~s}$ then used to serve female $F_{1} s$, resulted in Beeffalo having threeeighths blood from the American Bos gaurus and five-eighths from the Bos taurus. Males of this kind not only have typical hybrid characteristics, but also normal fertility. However, $F_{1} s$ with similar blood to the Beeffalo are still sterile. One important thing in this success in breeding, we think, is that the idea of gradual advance to fertility is applied in the experiments. The previously held cytogenetic theory of hybridization makes it impossible to explain this success.

Variety in hybrid sterility and the gradual progression to fertility can also be taken as a complement to the theory of how species have been formed. Taxonomy before the 1950s was based mainly on morphological characteristics and the existence of reproductive disturbance. Later, the findings of cytology, physiology, biochemistry, immunology, molecular biology and genetic engineering were introduced into taxonomy. The fusion, exchange and introduction of genetic materials of different species caused by genetic engineering may form microbes with new characteristics. If these new microbes are named as a new species, it is acceptable from the taxonomic point of view. Hybridization is also a matter of crosswise gene flow, and if hybrid sterility is not as absolute as people usually think, and that a few individuals among hybrids can produce germ cells to cause the appearance of new types or new species, it will not be so difficult to understand how a progressive advance to fertility can be achieved.

Among the eight $F_{1}$ animals which produced liveborn $B_{1}$ progeny, seven seemed to show that a mixture of horse and donkey chromosomes had been inherited via the egg. The proportional mix was, however, different in each individual.

It has not been possible to decide on the origin of each individual chromosome but some individual chromosomes do appear to be present in the somatic cells of different $B_{1} s$. In three $B_{1} s$ for example, the largest non-acrocentric pair could be recognized, this chromosome appearing the same on G-banding both in the horse and donkey (they can be distinguished by C-banding). Thus in $\mathrm{B}_{1} \mathrm{~s}$, there exists the possibility of these being of the same origin (both donkey or both horse) or of mixed origin. Thus, allodiploids may be a combination of chromosomes of the same origin, of different origin or of partially similar origin. Generally speaking, this phenomenon can only be found in hybrids, and in these allodiploids there will be chromosomes with partial or complete synapsis at meiosis. The success of such a mixed meiosis like this will probably be small but is not impossible. Since $F_{1}$ allodiploid mules and hinnies can produce eggs with chromosomes from both the horse and donkey, and their $B_{1}$ male progeny which are non-euploid allodiploids can also produce a few spermatozoa as shown in our study, 
then it could be possible to produce a new breed or species from hybridization.

\section{REFERENCES}

ANDERSON, w. S. 1939. Fertile mare mules. J. Hered., 30, 549-551.

BASRUR, P. K., BASRUR, V. R. AND GILMAN, J. P. W. 1963. A simple method for short term cultures from small biopsies. Exp. Cell Res., 30, 229-232.

BENIRSCHKE, K., BROWNHILL, L. E. ANID BEATH, M. M. 1962. Somatic chromosomes of the horse, the donkey and their hybrids, the mule and the hinny. J. Reprod. Fertil, 4, 319 . 326.

BENIRSCHKE, K. AND SULLIVAN, M. 1966. Corpora lutea in proven mules. Fertility and Sterility., 17, 24-33.

BENIRSCHKE, K., LOW, R. J., SULLIVAN, M. AND CARTER, R. M. 1964. Chromosome study of an alleged fertile mare mule. J. Hered., 55, 31-38.

BIELANSKI, W. AND ZAPLETAL. Z. 1968. Ovulation in shemules; a report of two cases. Proc. 6th Int. Cong. Animal Reprod. and Artificial Insemination, Paris, 2, 1555-1558.

CHANDLEY, A. C. 1981. Does 'affinity hold the key to fertility in the female mule'? Genet. Res. Camb., 37, 105-109.

CHANDLEY, A. C., JONES, R. C., DOTT, H. M., ALLEN, W. R. AND SHORT, R. V. 1974. Meiosis in interspecific equine hybrids. I. The male mule (Equus asinus $\times$ E. caballus) and hinny (E. caballus $\times$ E. asinus), Cytogenet. Cell Genet., 13, 330-341.

CHEN, T. R. 1974. A simple method to sequentially reveal Q and C-bands on the same metaphase chromosomes. Chromosoma, 47, 147-156.

CHENG GUANGCHAO, WANG; Ll, DING YURONG AND SU DONGDUEN, 1986. A primary observation of the chromosomes in somatic cells of $F_{3}$ from Cairina Moschata Domestica and Gaoyou Anas. Thesis for the Chinese National Scientific Seminar on Animal Hybrida, pp. 61-62.

CUÉNOT 1947. Species, translated by Zhou Taixuan. Commercial Press, 180-183.

DEKLENEV, E. N. 1984. The growth and reproductive factor of the hybrids from Java Bos gaurus and Bos taurus, abstracted and translated by Zhou Hongsun. Animal Science Digest., 4, 6.

ELDRIDGE, F. AND BLAZAK, W. F. 1976. Horse, ass, and mule chromosomes. J. Hered., 67, 361-367.

ELDRIDGiE, F. AND SUZUKI, Y. 1976. A mare mule-dam or foster mother? J. Hered., 67, 353-360.

IIARE, W. C. D. ANi SINGH, E. 1. 1979. Cytogenetics in Animal Reproduction, Commonwealth Agricultural Bureau.
KAMINSKI, M. AND GAJOS, F. 1964. Comparative examination of carboxylic esterases in sera of horse, donkey and their hybrids. Nature, 201, 716-718.

LI SHENGILI, LIU ANDE, CAI YOUYU, LIU YU, LI SHUHUA AND XU CHANGLONG 1980. The study of karyotypes of lymphocyte in peripheral blood from horse, ass, mules and hinnies. Thesis for the seminar on reproductive biology.

RICHER, C. L. AND ROMAGNANO, A. 1985. Cell synchronization and dynamic $\mathrm{G}$ banding of equine chromosomes by bromodeoxyuridine, $J$. Hered., 76, 375-376.

RONCi, R., CAI, H., YANG, X. AND WEI, J. 1985. Fertile mule in China and her unusual foal. J. Roy. Soc. Med., 78, 821-826.

RONG, R., CHANDIEY, A. C., SONG, I., MCBEATH, S., TAN, I'. P., BAl, Q. AND SPEED, R. M. 1988. A fertile mule and hinny in China. Cytogenet. Cell Genet., 47, 134-139.

RYDER, O. A., CHEMNICK, 1. G., BOWLING, A. T. AND BENIRSCHKE, K. 1985. Male mule qualifies as the offspring of a female mule and jack donkey. J. Hered., 76, 379-381.

RYDER, O. A., EPEL. N. C. ANI BENIRSCHKE, K. 1978. Chromosome banding studies of the Equidae. Cytogenet. Cell Genet., 20, 323-350.

SU DONGDUEN, DING YURONG AND ZHANG; YIXUN 1986. Observation of reproductivity of hybrids from Cairina Moschata Domestica and Gaoyou Anas. Thesis for the Chinese National Scientific Seminar on Animal Hybrida, pp. 58-60.

TAYLOR, M. J. AND SHORT, R. V. 1973. Development of the germ cells in the ovary of the mule and hinny. J. Reprod. Fert., 32, 441-445.

TRUJILLO, J. M., STENIUS, C., CHRISTIAN, L. AND OHNO, S 1962. Chromosomes of the horse, the donkey and the mule. Chromosoma, 13, 243-248.

TRUJILLO, J. M., OHNO, S., JARIDINE, J. H. AND ATKINS, N. B. 1969. Spermatogenesis in a male hinny: Histological and cytological studies. J. Hered., 60, 79-84.

ZHANG SONGZONG, CHEN RUEIXIAN, LAI YUANZHONG ANI) CHENG YIXIN 1986. Summary of the experiment for the application of hybrid vigor of hybrids between different species and genus of Duck. Thesis for the Chinese National Scientific Seminar on Animal Hybrida, pp. 47-57.

ZHENG LEYI, 1987. Principles and Methods for Animal Classification. High Education Press, Beijing, pp. 85-100.

ZONG ENZE 1954. Discussion of mules' reproductive problem based on the investigations of mules' fertility. Chinese Journal of Animal Husbandry and Veterinary Medicine Science, 1, 12-15.

ZONG ENZE, CHENG ZHONGXIAN AND LIU GUANGJIAO 1955. On mules' reproduction. Bulletin of Biology, 8, 11.

ZONG ENZE: 1983. The reproduction of $j u\left(B_{1}\right)$ born by female mules. Bulletin of Biology, 3, 17-19.

ZONG ENZE, FAN GENGQUAN, YIN HAIFU, WANG BAOLI, ZANG CAIFANG, SUN MINGJING AND JIAO SHUO 1985. A study on the chromosomes of interspecific $F_{2}$ hybrids between horse and ass. Scientia Agricultura Sinica, 1, 83-85. 